\title{
Aspectos Clínicos e Metabólicos de Mulheres na Pós-Menopausa Tratadas com Tibolona*
}

\author{
Clinical and Metabolic Aspects of Postmenopausal Women Treated with Tibolone
}

\author{
Salim Wehba ${ }^{1}$, Rogério Bonassi Machado ${ }^{1}$, César Eduardo Fernandes ${ }^{1}$, \\ José Arnaldo Souza Ferreira', Nilson Roberto de Melo $^{2}$, \\ José Mendes Aldrighi ${ }^{1}$, Edmund Chada Baracat ${ }^{3}$
}

\begin{abstract}
RESUM0
Objetivos: analisar a repercussão da tibolona sobre os sintomas do climatério e sobre algumas variáveis clinicas e metabólicas.

Métodos: foram avaliadas 34 pacientes na pós-menopausa que foram submetidas ao tratamento com tibolona na dose de 2,5 mg/dia por 48 semanas. Foram analisados os seguintes parâmetros: peso, pressão arterial e perfis lipidico e lipoprotéico, representados pelos niveis de colesterol total, $H D L-c, L D L-c$, VLDL-c e triglicerideos.

Resultados: melhora relevante da sintomatologia do climatério foi demonstrada por decréscimo significativo do indice menopausal de Kupperman $(p<0.001)$ e da freqüência média das ondas de calor ( $p<0.001)$. Estes efeitos foram observados já no primeiro mês de tratamento, mantendo-se até o final do estudo. Houve decréscimo significante nos niveis de colesterol total, triglicerideos e VLDL-c ( $p<0.001)$. Os niveis de $L D L-c$ apresentaram discreta redução (não-significante). Os niveis de HDL-c mostraram decréscimo significante na $24^{a}$ semana de tratamento, mas retornaram aos niveis basais na $48^{a}$ semana. Não foram observadas modificações significantes no peso e pressão arterial. Os efeitos colaterais relatados incluiram sangramento vaginal, náusea e edema, todos temporários e de intensidade leve.

Conclusão: a tibolona representa uma opção para o tratamento dos sintomas climatéricos em mulheres na pós-menopausa, sem alterações relevantes no perfil lipídico e lipoprotéico.
\end{abstract}

PALAVRAS-CHAVE: Terapia de reposição hormonal. Perfil lipídico. Climatério.

\section{Introdução}

O alivio da sintomatologia climatérica, bem como a redução do risco cardiovascular têm sido há tempos atribuídos à terapêutica de reposição hormonal (TRH) no climatério, propiciando melhora significativa na qualidade de vida das mulheres nesse período.

A administração de estrogênios, largamente utilizados em todo o mundo, determina atenua-

\footnotetext{
1 - Departamento de Obstetrícia e Ginecologia da Faculdade de Ciências Médicas da Santa Casa de São Paulo

2 - Clínica Ginecológica da Faculdade de Medicina da Universidade de São Paulo

3 - Disciplina de Ginecologia da UNIFESP - Escola Paulista de Medicina

Correspondência:

Salim Wehba

Av. Indianópolis, 2700

04062-003 - São Paulo - SP
}

ção da sintomatologia vasomotora, representada particularmente pelas ondas de calor ${ }^{1}$, além de atuar de maneira significante no trofismo urogenital, metabolismo ósseo, sistema cardiovascular e sistema nervoso central ${ }^{2,3,4,5}$.

O metabolismo lipídico e lipoprotéico sofre alterações ao longo da vida, notadamente após a ocorrência da menopausa, traduzindo-se em perfil de risco cardiovascular. A reposição estrogênica, por sua vez, tem demonstrado normalizar as alterações metabólicas desse período da vida da mulher, o que, somado à ação vascular desse esteróide, determina redução na mortalidade pela doença cardiovascular nas usuárias dessa modalidade terapêutica ${ }^{6,7,8}$.

Entretanto, o número de usuárias de TRH permanece aquém do esperado. A falta de aderência ao tratamento é também bastante comum: 40 a $60 \%$ das mulheres suspendem o tratamento 
antes do primeiro ano e apenas 5 a 34\% mantêm a TRH por cinco anos ${ }^{9}$. A presença de sangramento e sintomas semelhantes à tensão pré-menstrual, particularmente mastalgia, eventos amiúde observados durante estrogenioterapia isolada ou em combinação aos progestagênios, são os motivos mais comuns para a descontinuidade do tratamento ${ }^{10}$.

Dessa maneira, o emprego de novos compostos tem sido aventado, na tentativa de minimizar os efeitos indesejáveis da estrogenioterapia de reposição. A tibolona, esteróide sintético derivado do noretinodrel, apresenta características farmacocinéticas singulares, que incluem atividade estrogênica, progestagênica e androgênica $^{11}$, sendo utilizado em esquema contínuo para terapêutica de reposição hormonal. Aliando o alívio sintomatológico à estabilidade endometrial, o uso da tibolona parece representar alternativa atraente em TRH em mulheres na pós-menopausa.

O presente estudo tem por objetivo analisar parâmetros clínicos, com ênfase na sintomatologia climatérica, e metabólicos, representados pelo perfil lipídico e lipoprotéico, em mulheres na pósmenopausa submetidas ao tratamento com tibolona.

\section{Pacientes e Métodos}

Foram avaliadas 34 mulheres com sintomatologia decorrente da síndrome menopausal em estudo aberto, não-comparativo, submetidas ao tratamento com tibolona na dose de $2,5 \mathrm{mg} /$ dia continuamente, por 48 semanas, de acordo com protocolo clínico estabelecido. Este previa a inclusão de pacientes saudáveis no período pós-menopausal com idade entre 40 e 70 anos, que apresentassem útero íntegro, tempo de pós-menopausa de no mínimo 1 ano, niveis de gonadotrofinas compativeis com os valores observados em mulheres menopausadas e mamografia sem sinais de malignidade há no mínimo 12 meses.

Foram excluídas pacientes com as seguintes condições médicas concomitantes: endométrio com espessura maior que $5 \mathrm{~mm}$ à ultra-sonografia transvaginal; utilização de estrogênios, progestagênios ou outro esteróide nos últimos 3 meses; sangramento vaginal anormal não diagnosticado dentro de 12 meses antes da seleção; neoplasia estrogênio-dependente; distúrbios tromboembólicos ou distúrbios hepático ou renal graves. O protocolo de estudo foi previamente aprovado pelas Comissões de Ética em Pesquisas da Santa Casa de São Paulo, do Hospital das Clínicas da Univer- sidade de São Paulo e da Universidade Federal de São Paulo.

Selecionada a paciente, realizava-se anamnese, com ênfase na abordagem dos sintomas climatéricos pelo índice de Kupperman ${ }^{12}$, exames físico e ginecológico completos, avaliação ou solicitação do exame colpocitológico cervicovaginal e da mamografia e dosagens de colesterol total, LDLcolesterol (LDL-c), HDL-colesterol (HDL-c), VLDLcolesterol (VLDL-c), triglicerídeos. Era também obtida a anuência da paciente após consentimento livre e esclarecido.

Incluída a paciente, iniciava-se o tratamento com tibolona por via oral, na dose de $2,5 \mathrm{mg}$ diariamente. Retornos a cada 4 semanas foram realizados, observando-se atentamente as queixas relacionadas, eventos adversos e alterações clínicas relevantes.

A análise dos parâmetros clínicos constava de aferição da pressão arterial e peso corpóreo, exame ginecológico, avaliação dos sintomas menopausais pela escala de Kupperman e verificação da ocorrência de eventos adversos e/ou medicações concomitantes.

Novas dosagens de colesterol total, LDL-c, HDL-c, VLDL-c e triglicerídeos foram realizadas ao final da 24a e da 48 semanas de tratamento.

$\mathrm{Na}$ avaliação da eficácia terapêutica foram analisados:

- a evolução dos sintomas da menopausa (sintomas vasomotores, parestesia, distúrbios do sono, irritabilidade, ansiedade, depressão, vertigem, fadiga, artralgia, mialgia, cefaléia, palpitação, vagina seca, prurido, corrimento e urgência miccional), utilizando-se a graduação: $0=$ ausente; 1 = leve; 2 = moderado e 3 = intenso.

- o índice de Kupperman (indice menopáusico), obtido pela soma dos produtos das respectivas graduações dos sintomas da menopausa acima citadas, pelos seguintes fatores de conversão: sintomas vasomotores $(\mathrm{x} 4)$, parestesias $(\mathrm{x} 2)$, distúrbios do sono (x2), irritabilidade (x2), depressão (x1), vertigem $(\mathrm{x} 1)$, fadiga $(\mathrm{x} 1)$, artralgia/mialgia $(\mathrm{x} 1)$, cefaléia (x1) e palpitação $(\mathrm{x} 1)$.

- o número de fogachos por dia, utilizando a escala de até 5 vezes, de 6 a 10 e acima de 10 vezes ao dia, obtendo-se então as médias das 34 pacientes estudadas.

A Tabela 1 representa as médias da idade, tempo de pós-menopausa e as características clínicas, como peso e pressão arterial. Em apenas 1 caso $(2,9 \%)$ a causa da menopausa foi cirúrgica (ooforectomia bilateral). Quanto ao grupo étnico, $88,2,8,8$ e $2,9 \%$ das pacientes eram constituídas pelas raças branca, parda e negra, respectivamente. 
Tabela 1 - Características clínicas de 34 mulheres na pós-menopausa, antes do tratamento com tibolona (média \pm desvio padrão).

\begin{tabular}{lc}
\hline Idade (anos) & $51,8 \pm 5,7$ \\
Tempo de menopausa (anos) & $4,7 \pm 4,0$ \\
Peso (kg) & $66,9 \pm 13,3$ \\
Pressão arterial sistólica (mmHg) & $128,8 \pm 13,4$ \\
Pressão arterial diastólica (mmHg) & $83,4 \pm 8,6$ \\
\hline
\end{tabular}

Por se tratar de um estudo não-comparativo, aplicou-se a estatística descritiva para as características demográficas e os antecedentes ginecológicos da amostra, e análise da variância para as comparações da evolução de sinais e sintomas decorrentes da menopausa, com nivel de significância fixado em $5 \%$.

\section{Resultados}

Uma redução importante do índice de Kupperman, com significância estatística, foi observada após o primeiro mês, notando-se queda progressiva ao longo de todo o tratamento com tibolona. Os escores do índice menopausal apresentavam redução significativa na 4a semana de tratamento e em 48 semanas apresentavam níveis médios de 2,1 . Ao se considerar isoladamente as ondas de calor, representadas pela freqüência dos fogachos ao dia, também observamos redução estatisticamente significante já nas primeiras 4 semanas, mantendo-se estáveis durante todo o tratamento.

Ocorreu decréscimo significativo dos níveis de colesterol total, triglicerídeos e VLDL-colesterol, observado após 24 semanas de tratamento, e que se manteve até o final do estudo. Os níveis de LDLcolesterol apresentaram discreta redução (sem significância estatística). Já os niveis de HDLcolesterol mostraram-se significativamente menores na $24^{\mathrm{a}}$ semana, retornando porém, aos valores próximo dos basais, mas ainda menores, após 48 semanas de tratamento com tibolona (Tabela 2).

Em relação aos parâmetros clínicos estudados, demonstrou-se discreta elevação média no peso corporal ao longo do tratamento, sem significância estatística. Não foram observadas alterações no comportamento dos niveis pressóricos sistólico e diastólico médios durante as 48 semanas do seguimento em usuárias de tibolona, como mostra a Tabela 3 .

Os efeitos colaterais observados foram de intensidade leve e temporários, correspondendo na maioria das vezes a um discreto sangramento vaginal (4 casos), náuseas e edema.
Tabela 2 - Níveis de triglicerídeos, colesterol total, LDL-c, HDL-c e VLDL-c, em mg\%, antes e após 24 e 48 semanas de tratamento com tibolona (M $\pm \mathrm{DP})$.

\begin{tabular}{lccc}
\hline & Pré-tratamento & 24 semanas & 48 semanas \\
\hline Triglicerídeos & $129.4 \pm 61,6$ & $87,7 \pm 40,4^{* *}$ & $96,8 \pm 42^{* *}$ \\
Colesterol total & $232.2 \pm 40,6$ & $209,6 \pm 44,7^{*}$ & $216,9 \pm 41^{*}$ \\
LDL-C & $165.6 \pm 36,5$ & $163,5 \pm 44,2$ & $159,0 \pm 40,5$ \\
HDL-C & $40.1 \pm 9,2$ & $28,8 \pm 10,9^{* *}$ & $38,7 \pm 14,7^{* *}$ \\
VLDL-C & $26.5 \pm 12,6$ & $17,2 \pm 8^{* *}$ & $20,0 \pm 9,5^{* *}$ \\
\hline
\end{tabular}

${ }^{*} p<0.01$

** $p<0.001$

${ }^{\star * *} p<0.001$ (24 semanas $x 48$ semanas)

LDL-C - colesterol de baixa densidade HDL-C - colesterol de alta densidade VLDL-C - colesterol de muito baixa densidade

Tabela 3 - Evolução do peso corporal (kg) e da pressão arterial sistólica (PAS) e diastólica (PAD) em 34 pacientes na pós menopausa, antes e após 24 e 48 semanas de tratamento com tibolona $(\mathrm{M} \pm \mathrm{DP})$.

\begin{tabular}{lcrr}
\hline & Pré-tratamento & 24 semanas & 48 semanas \\
\hline Peso corporal $(\mathrm{kg})$ & $66,9 \pm 13,3$ & $68,6 \pm 12,9$ & $68,1 \pm 12,1$ \\
PAS $(\mathrm{mmHg})$ & $128,8 \pm 13,4$ & $127,6 \pm 15,3$ & $126,1 \pm 12,2$ \\
PAD $(\mathrm{mmHg})$ & $83,4 \pm 8,6$ & $80,6 \pm 7,5$ & $84,1 \pm 7,3$ \\
\hline
\end{tabular}

\section{Discussão}

A análise da presente casuística demonstrou que a administração de tibolona em dose diária de $2,5 \mathrm{mg}$ foi efetiva no alivio dos sintomas climatéricos, representado pela redução significativa do índice menopausal total e, isoladamente, na freqüência média das ondas de calor durante o tratamento. Cumpre assinalar que os efeitos ocorreram a curto prazo, objetivo primordial em TRH, que na maioria das vezes traduz-se em maior aceitação e entusiasmo pela continuidade do tratamento. Também são responsáveis por maior aderência à TRH a ausência de eventos adversos, em particular relacionados ao sangramento inesperado, que nesse estudo se mostraram com baixa incidência (4 casos), de leve intensidade, não necessitando de tratamento adicional. Os resultados observados, embora não se tratando de estudo comparativo, foram corroborados por outros autores, demonstrando a eficácia da tibolona na redução dos sintomas climatéricos ${ }^{13,14,15,16}$.

Observamos que não ocorreu alteração dos niveis pressóricos e ponderais após 48 semanas de tratamento com tibolona, também em concordância com a literatura pertinente ${ }^{15}$.

A análise dos lipídeos e lipoproteínas teve por objetivo determinar a influência da tibolona nesses marcadores da doença cardiovascular. Sabe-se que o fenômeno aterosclerótico decorre do desequilíbrio entre fatores de coagulação e 
fibrinólise, resultando na formação de trombo, com posterior obstrução vascular. A iniciação desse processo, no entanto, baseia-se na formação da placa ateromatosa, com a deposição vascular de lipídeos e lipoproteínas e posterior lesão endotelial ${ }^{17}$. A relação inversa entre os niveis da lipoproteína de alta densidade (HDL-c) e o risco cardiovascular tem sido há tempos observada ${ }^{18}$, bem como a participação da lipoproteína de baixa densidade (LDL-C), apolipoproteínas e lipoproteína(a) como fatores de risco para a doença cardiovascular ${ }^{19,20}$. A elevação dos triglicerídeos, comumente observada com o uso de estrogênios, por determinar aumento nos niveis de LDL-c, pode representar risco adicional para a formação da placa ateromatosa, constituindo fator de risco isola$\mathrm{do}^{21}$.

A utilização da tibolona em nosso estudo não se refletiu em piora no padrão lipídico e lipoprotéico. A redução nos niveis de colesterol total, triglicerídeos e VLDL-c, mantida até o final do tratamento, são concordantes com os achados de outros autores ${ }^{15,22,23}$. Foi observado um decréscimo nos niveis de HDL-c, possivelmente relacionado ao perfil hormonal misto da tibolona, que inclui ações estrogênicas, progestagênicas e androgênicas. Embora esse achado tenha sido previamente descrito, por vezes tem representado motivo de controvérsias a respeito da atuação cardiovascular da tibolona ${ }^{15}$. Por outro lado, os níveis de LDL-c, fator de risco independente para a doença cardiovascular, apresentaram decréscimo significante, mantendo-se estáveis até o final do tratamento.

Sabe-se que para a prevenção da doença cardiovascular, além de perfis lipídico e lipoprotéico favoráveis, tem importância relevante o impacto do hormônio utilizado sobre os fatores de coagulação e fibrinólise. Dados atuais demonstram melhora do perfil antitrombogênico com o uso da tibolona, equilibrando dessa forma a possivel redução nos niveis do HDL-c ${ }^{22,23}$.

Rotineiramente, a avaliação do risco cardiovascular em mulheres na pós-menopausa é realizada por meio de parâmetros clínicos e, laboratorialmente, pelo perfil lipídico e lipoprotéico. A dosagem de lipoproteina(a), assim como o acesso a marcadores específicos da coagulação e fibrinólise nem sempre são factiveis, estando na maior parte das vezes distantes da prática diária do médico. Assim, a combinação de fatores de risco clínicos, dosagens laboratoriais de rotina parece ser razoável, podendo-se instituir esquemas de TRH com segurança. Em nosso estudo utilizamos dosagens rotineiras, que em combinação com dados clínicos demonstram bom perfil metabólico em usuárias de tibolona.

\section{SUMMARY}

Purpose: to evaluate the effects of tibolone on climacteric symptoms and clinical and metabolic variables.

Methods: thirty-four postmenopausal women were treated orally with $2.5 \mathrm{mg}$ tibolone daily for 48 weeks and evaluated as to climacteric complaints, clinical aspects such as weight and blood pressure and lipid profile (total cholesterol, $H D L$ $c, L D L-c, V L D L-c$ and triglycerides).

Results: a significant improvement of climacteric complaints was demonstrated by a significant decrease in the Kupperman index $(p<0.001)$ and the mean number of hot flushes $(p<0.001)$ from the first month of treatment onwards. There was a significant decrease in total cholesterol, triglycerides and VLDL-c $(p<0.001)$. The LDL-c levels presented a slight decrease (not significant). The HDL-c levels showed a significant decrease at week 24. However these levels returned to baseline levels at week 48 . With regard to the vital signs no change in body weight and blood pressure was measured. The side effects were mild and temporary, vaginal bleeding, nausea and edema being the most common.

Conclusion: tibolone may be considered a safe and efficient option to treat climacteric symptoms in postmenopausal women without significant impact on lipid profile.

KEY WORDS: Hormonal replacement therapy. Lipid profile. Climacterium.

\section{Referências}

1. Ravnikar V. Physiology and treatment of hot flushes. Obstet Gynecol 1990; 75 Suppl:3S-8S.

2. Lauritzen C. Clinical use of oestrogens and progestogens. Maturitas 1990;12:199-214.

3. Stampfer MJ, Colditz GA. Estrogen replacement therapy and coronary heart disease: a quantitative assessment of the epidemiologic evidence. Prev Med 1991; 20:47-63.

4. Henderson VW, Paganini-Hill A, Emanuel CK, Dunn $\mathrm{ME}$, Buckwalter JG. Estrogen replacement therapy in older women. Comparisons between Alzheimer's disease cases and nondemented control subjects. Arch Neurol 1994;51:896-900.

5. Tang MX, Jacobs D, Stern Y, Marder K, Schofield P, Gurland B, Andrews H, Mayeux R. Effect of oestrogen during menopause on risk and age of at onset Alzheimer's Disease. Lancet 1996;348:429-32.

6. Lobo RA. Cardiovascular implications of estrogen replacement therapy. Obstet Gynecol 1990;75 Suppl:18S-25S. 
7. Henderson BE, Paganini-Hill A, Ross RK. Decreased mortality in users of estrogen replacement therapy. Arch Intern Med 1991;151:75-8.

8. Stampfer MJ, Colditz GA, Willett WC, Manson JE, Rosner B, Speizer FE, et al. Postmenopausal estrogen therapy and cardiovascular disease: 10 year follow-up from the Nurse's Health Study. N Engl J Med 1991;325:756-62.

9. Speroff T, Dawson NV, Speroff L, Haber RJ. A riskbenefit analysis of elective bilateral oophorectomy: effect of changes in compliance with estrogen therapy on outcome. Am J Obstet Gynecol 1991;164:165-74.

10.Ravnikar VA. Compliance with hormone therapy. Am J Obstet Gynecol 1987;156:1332-4.

11.De Visser J, Coert A, Feenstra H, van der Viers J. Endocrinological studies with (7 alpha, 17 alpha)17-hydroxy-7-methyl-19-norpregn-5(10)-en-20yn-3-one (Org OD 14). Arzneimittelforschung 1984;34:1010-7.

12.Kupperman HS, Blatt MHG, Wiesbaden H, Fuller W. Comparative clinical evaluation of estrogen preparations by menopausal and amenorrheal indices. J Clin Endocrinol 1953;13:688-94.

13.Volpe A, Facchinetti F, Grasso A, Petraglia F, Camparini D, Genazzani AR. Benefits and risks of different hormonal replacement therapies in postmenopausal women. Maturitas 1986;8:327-34.

14.Crona N, Samsioe G, Lindberg UB, Silferstolpe G. Treatment of climacteric complaints with Org OD 14: a comparative study with oestradiol valerate and placebo. Maturitas 1988;9:303-8.

15.Ginsburg J, Prelevic G, Butler, D, Okolo S. Clinical experience with tibolone (Livial) over 8 years. Maturitas 1995;21:71-6.
16.Egarter C, Huber J, Leikermoser R, Haidbauer R, Pusch H, Fischl F, et al. Tibolone versus sequential conjugated estrogens and progestin in the treatment of climateric complaints. Maturitas 1996;23:55-62.

17.Godsland IF, Wynn V, Crook D, Miller NE. Sex, plasma lipoproteins, and atherosclerosis; prevailing assumptions and outstanding questions. Am Heart J 1987;114:1467-503.

18. Castelli WP, Garrison RJ, Wilson PW, Abbott RD, Kalousdian $\mathrm{S}$, Kannel WB. Incidence of coronary heart disease and lipoprotein cholesterol levels. The Framingham Study. JAMA 1986;256:2835-8.

19. Maciejko JJ, Holmes DR, Kottke BA, Zinsmeister AR, Dinh DM, Mao SJ. Apolipoprotein A-1 as a marker of angiographically assessed coronary-artery disease. N Engl J Med 1983; 309:385-9.

20.Dahlen GH. Lp (a) lipoprotein in cardiovascular disease. Atherosclerosis 1994;108:111-26.

21.Walsh BW, Schiff I, Rosner B, Greenber L, Ravnikar V, Sacks FM. Effects of postmenopausal estrogen replacement on the concentrations and metabolism of plasma lipoproteins. N Engl J Med 1991;325:1196-204.

22.Rosati D, Micheli S, Pinto S, Fedi S, Bruni V, Bucciantini $\mathrm{S}$, et al. Changes in lipidic and hemostatic parameters induced by tibolone treatment. Thromb Res 1997;85:273-8.

23.Bjarnason NH, Bjarnason K, Haarbo J, Bennink HJ, Christiansen $\mathrm{C}$. Tibolone: the influence on markers of cardiovascular disease. J Clin Endocrinol Metab 1997;82:1752-6. 